\title{
Circadian Regulation of cGMP-Gated Channels of Vertebrate Cone Photoreceptors: Role of cAMP and Ras
}

\author{
Gladys Y.-P. Ko, Michael L. Ko, and Stuart E. Dryer \\ Department of Biology and Biochemistry and Biological Clocks Program, University of Houston, Houston, Texas 77204-5001
}

\begin{abstract}
Circadian oscillators in chicken cone photoreceptors regulate the gating properties of cGMP-gated cationic channels (CNGCs) such that they have a higher apparent affinity for cGMP during the subjective night. Here we show that cAMP, acting through protein kinase A (PKA), Ras, and Erk, is part of the circadian output pathway controlling CNGCs. Endogenous and exogenous cAMP cause activation of Erk and Ras, which are more active at night in cones, and increase the apparent affinity of CNGCs for cGMP. The Ras farnesyl transferase inhibitor manumycin-A, and a dominant-negative form of Ras (RasN17) block the circadian rhythms in CNGC gating, as well as the effects of cAMP. A dominant-negative form of the MEK kinase B-Raf also blocks circadian and cAMP modulation of CNGCs. The circadian output pathway modulating CNGC channels is comprised in part of cAMP $\rightarrow$ PKA $\rightarrow$ Ras $\rightarrow$ B-Raf $\rightarrow$ MEK $\rightarrow$ Erk $\rightarrow \rightarrow$ CNGCs. cAMP activation of Ras and Erk occur within minutes, whereas modulation of CNGCs requires $>1 \mathrm{hr}$. However, cAMP protagonists do not alter rhythms in cPer2 mRNA, and their effects on CNGCs cannot be attributed to clock phase-shifting.
\end{abstract}

Key words: circadian; phototransduction; ERK; Ras; cAMP; channel; Raf

\section{Introduction}

A large literature indicates that circadian oscillators regulate vertebrate visual system function (Terman and Terman, 1985; Bassi and Powers, 1987; Dearry and Barlow, 1987; Reme et al., 1991; Shaw et al., 1993; Lu et al., 1995; Wang and Mangel, 1996; Li and Dowling, 1998; Manglapus et al., 1998, 1999; McGoogan and Cassone, 1999; Wu et al., 2000). Control of visual system sensitivity is associated with several rhythmic changes in the structure and physiology of the retina and associated ocular structures (Cahill and Besharse, 1995). However, relatively little is known about circadian control of individual components of phototransduction cascades.

We recently reported that circadian oscillators within photoreceptors modulate chick cone cGMP-gated channels (CNGCs) (Ko et al., 2001, 2003), which carry the photoreceptor dark current (Burns and Baylor, 2001). Thus, the apparent affinity of CNGCs for cGMP is significantly greater during the subjective night than during the subjective day, even in constant dark conditions, such that considerable changes in channel gating would be expected to occur at physiological concentrations of cGMP (Ko et al., 2001, 2003). Light exposure in and of itself does not cause modulation of these channels, and other biophysical features of the gating of CNGCs, such as unitary conductance, Hill

Received July 30, 2003; revised Dec. 11, 2003; accepted Dec. 15, 2003.

This work was supported by National Institutes of Health (NIH) Grant EY11973 to S.E.D. and NIH postdoctoral fellowship EY13920 to G.Y.-P.K. We thank Drs. Deborah Morrison (National Cancer Institute, Bethesda, MD), Johannes Bos (University Medical Center of Utrecht, Utrecht, The Netherlands), and Phillip Stork (Vollum Institute, Portland, $\mathrm{OR}$ ) for providing Raf and Rap constructs.

Correspondence should be addressed to Dr. Stuart E. Dryer, Department of Biology and Biochemistry, University of Houston, Houston, TX 77204-5001. E-mail: Sdryer@uh.edu.

DOI:10.1523/JNEUROSCI.3560-03.2004

Copyright $\odot 2004$ Society for Neuroscience $\quad$ 0270-6474/04/241296-09\$15.00/0 slope, or density of channels in the plasma membrane, do not vary significantly as a function of the time of day (Ko et al., 2001).

Circadian modulation of CNGCs entails a post-translational modification of the channel complex, and it qualitatively resembles effects that others have observed after binding of calmodulin and related proteins, or phosphorylation on specific CNGC tyrosine residues (Rebrik and Korenbrot, 1998; Kramer and Molokanova, 2001; Molokanova and Kramer, 2001). Modulation of photoreceptor CNGCs is predicted to evoke complex effects on the dynamics of phototransduction (Pugh et al., 1999; Savchenko et al., 2001). Moreover, because active CNGCs in cones also contribute to $\mathrm{Ca}^{2+}$ dependent exocytotic secretion of neurotransmitter (Rieke and Schwartz, 1994; Savchenko et al., 1997), modulation of CNGCs may also regulate light responses of second-order retinal cells.

Circadian modulation of cones is driven in part by rhythms in the activity of the MAP kinase Erk. Thus, Erk activation in cones exhibits a robust circadian rhythm and peaks around the middle of the subjective night. This rhythm is part of the output pathway leading to modulation of CNGCs, because inhibition of the Erk signaling cascade blocks the circadian rhythm in the gating properties of CNGCs (Ko et al., 2001). The components of the output pathway leading from the photoreceptor circadian oscillator to activation of Erk are the subject of the present study. Here we show that Erk activation, and therefore modulation of CNGCs, is driven by intracellular cAMP, which acts at least in part via protein kinase A (PKA) to activate a cascade comprised of Ras, B-Raf, and MEK.

\section{Materials and Methods}

Cell isolation and culture. Chick retinas were dissociated at embryonic day 6 (E6) as described previously (Adler and Hatlee, 1989; Ko et al., 2001, 2003). Retinal cells were grown for $5 \mathrm{~d}$ on poly-D-lysine-coated glass coverslips in a medium consisting of Eagle's minimal essential medium 
(Biowhittaker, Walkersville, MD) supplemented with 10\% heatinactivated horse serum (Biowhittaker), $2 \mathrm{~mm}$ glutamine, $50 \mathrm{U} / \mathrm{ml}$ penicillin, $50 \mu \mathrm{g} / \mathrm{ml}$ streptomycin, and $20 \mathrm{ng} / \mathrm{ml}$ recombinant rat ciliary neurotrophic factor ( $\mathrm{R} \& \mathrm{D}$ Systems, Minneapolis, MN). Cultures prepared in this way yield a highly enriched population of cones (Adler et al., 1984; Belecky-Adams et al., 1996). Cell culture incubators $\left(39^{\circ} \mathrm{C}\right.$ and $5 \%$ $\mathrm{CO}_{2}$ ) were equipped with lights and timers, which allowed for entrainment of retinal circadian oscillators to $12 \mathrm{hr}$ light/dark (LD) cycles in vitro or in ovo as described previously (Ko et al., 2001, 2003). Most measurements were made on the second day of constant darkness (DD), after $4 \mathrm{~d}$ of entrainment to LD cycles.

Electrophysiology. Recordings were made from cells with elongated cell bodies, an outer segment, and one or more prominent oil droplets on the distal side of the soma, as described in detail elsewhere (Ko et al., 2001, 2003). Briefly, inside-out patches were excised into a saline free of divalent cations consisting of (in mM): $145 \mathrm{NaCl}, 10 \mathrm{Na}$-HEPES, 10 glucose, and 1 EGTA, pH 7.4, and held at $-65 \mathrm{mV}$. Pipette solution was the same as the bath saline. Recordings were performed in the light at room temperature $\left(22-23^{\circ} \mathrm{C}\right)$. Channels were activated by gravity-fed bath application of varying concentrations of cGMP dissolved in bath saline. Cultures were typically pretreated with drugs at circadian time (CT) 3 or CT15 for $2 \mathrm{hr}$ or $15 \mathrm{~min}$ before recording, as indicated. Drug treatment occurred in the dark in the cell culture incubator. Concentration-response curves were fitted with the Hill equation $I_{\mathrm{s}}=I_{\mathrm{Max}}\left[S^{n} /\left(K_{\mathrm{D}}{ }^{n}+\right.\right.$ $\left.S^{n}\right)$ ] where $S$ is the concentration of cGMP, $K_{\mathrm{D}}$ is the dissociation constant, and $n$ is the Hill coefficient using Microcal (Northampton, MA) Origin version 6.0 software. Each group contained 9-12 patches obtained from at least three different preparations of retinal cells. All statistical analyses were performed using Statistica software (Statsoft, Tulsa, OK) and consisted of one-way ANOVA followed by Tukey's post hoc test for unbalanced $n$ (when comparisons were made between multiple independent groups). Throughout, $p<0.05$ was regarded as significant. The cAMP analog 8-CPT-cAMP and the adenylate cyclase activator forskolin were obtained from Sigma (St. Louis, MO); the adenylate cyclase inhibitors MDL-12330A and SQ-22536, and the farnesyl transferase inhibitor manumycin-A were obtained from Calbiochem (La Jolla, CA). The PKA inhibitor Rp-cAMPS was obtained from Tocris (Ballwin, MO), and the PKA inhibitor myristoyl-PKI ${ }_{\text {[14-22] }}$ was obtained from Biosource International (Camarillo, CA).

Biolistic transfection. Chick retinas from E6 were dissociated, cultured, and entrained under LD cycles for $4-5 \mathrm{~d}$ as described above in culture medium as described above except that horse serum was increased from 10 to $15 \%$. On the fourth or fifth day of culture, cells were transfected using a biolistic particle delivery system (PSD-1000; Bio-Rad, Hercules, $\mathrm{CA}$ ). Plasmids were precipitated onto $1.0 \mu \mathrm{m}$ gold beads according to the protocol of the company. The particle delivery system generated a helium shock wave with a pressure gradient of 650 psi to accelerate the coated beads onto cultured cells. After transfection, cells were cultured under DD for another day, and then used for electrophysiology at CT4-7 or CT16-19 on the second day of DD. The plasmid encoding modified Renilla reniformis green fluorescent protein (GFP) is commercially available from Stratagene (La Jolla, CA) and was chosen because it produces much less toxicity than Aqueorus green fluorescent protein. In these experiments, dominant-negative mutants were cotransfected with Renilla GFP at a ratio of 1:1. A plasmid encoding RasS17N (RasN17) was obtained from Upstate Biotechnology (Lake Placid, NY). Plasmids encoding the mutants B-Raf-km and Raf-1-kd were generously provided by Dr. Deborah Morrison of the National Cancer Institute (Bethesda, MD). Both of these constructs contain mutations in the ATP binding site that cause them to act as powerful dominant negatives. Constructs encoding RapN17, originally developed by Dr. Johannes Bos (University Medical Center of Utrecht, The Netherlands), were provided by Dr. Phillip Stork (Vollum Institute, Portland, OR). All of these constructs use cytomegalovirus (CMV) promoters.

Immunoblot analysis of protein kinase phosphorylation and Ras activation. Measurements of Erk activation by immunoblot analysis have been described in detail previously (Ko et al., 2001, 2003). We used a monoclonal antibody specific for diphospho-Erk (Sigma), and a polyclonal antibody insensitive to the phosphorylation state of Erk (Santa Cruz
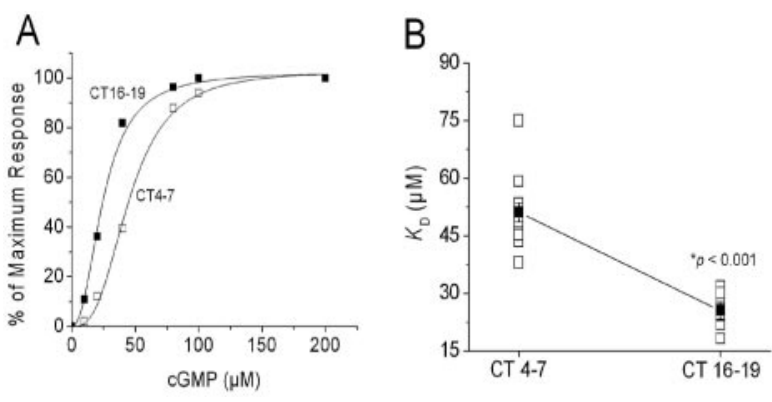

Figure 1. The apparent affinity of cGMP-gated channels for activating ligand in chick retinal cone photoreceptors is under circadian control. Cultured chick cones were entrained to $12 \mathrm{hr}$ LD cycles for $4-5$ din vitro and then switched to constant darkness (DD). On the second day of DD, inside-out patches were excised from cones during the subjective day (CT4-7) or the subjective night (CT16-19). cGMP concentration-response curves were generated immediately after patch excision. A, Typical CGMP concentration-response curves obtained from patches excised during the subjective day (hollow squares) and subjective night (filled squares). Curves are shown with superimposed least-squares fits to the Hill equation. $B$, Mean apparent $K_{D}$ (filled squares) from patches excised at CT4-7 ( $n=10$ patches) and (T16 -19 ( $n=10$ patches). Individual data points are also shown (open squares). Recordings were made from cells free running on the second day of $D D$. In this and all subsequent figures, error bars represent SEM, and numbers in parentheses are the number of patches tested. ${ }^{*} p<0.001$.

Biochemicals, Santa Cruz, CA). All experiments were repeated three to six times. Ras activation was assayed using a commercially available pulldown assay (Upstate Biotechnology) according to the manufacturer's instructions. This system uses the Ras binding domain (RBD) of Raf-1 to selectively precipitate active Ras, which is then measured by immunoblot analysis using a primary antibody directed against Ras. A polyclonal antibody against Rap1 was also obtained from Upstate.

Quantitative real-time reverse transcription-PCR analysis of cPer 2 transcripts. These procedures and all of the primers were described in detail previously (Ko et al., 2003). Briefly, total RNA from cultured chick retina was extracted, and $500 \mathrm{ng}$ of total RNA from each sample was used to quantify expression of $c$ Per 2 and chick $\beta$-actin mRNA by quantitative real-time RT-PCR (Q-PCR) using the Taqman one-step RT (reverse transcription)-PCR kit and an ABI Prism 7000 Sequence Detection System (Applied Biosystems, Foster City, CA). Data are expressed as the relative ratio of $c$ Per 2 to $\beta$-actin, and measurements were repeated three times.

\section{Results}

\section{cAMP contributes to circadian modulation of cone CNGCs}

Chick photoreceptors were entrained to $12 \mathrm{hr}$ LD cycles for $4-5 \mathrm{~d}$ in vitro, and then switched to constant darkness (DD). On the second day of $\mathrm{DD}$, inside-out patches were excised from cones during the subjective day, at CT4-7, or during the subjective night (at CT16-19). CT0 is the time of expected lights on, and CT12 is the time of expected lights off. cGMP concentrationresponse curves were determined immediately after patch excision (Fig. 1A). As described previously (Ko et al., 2001, 2003), the average apparent $K_{\mathrm{D}}$ for cGMP estimated from fitted Hill curves was significantly greater in patches excised during the subjective day than during the subjective night (Fig. $1 B$ ). Therefore, the affinity of CNGCs for their normal activating ligand is under circadian control in chicken cones and is higher during the night. As noted previously, circadian oscillators do not appear to modulate the Hill slope for channel activation or the average channel density (Ko et al., 2001).

In chick pineal photoreceptors, the activity of adenylate cyclase and the content of cAMP are under circadian control and peak during the middle of the subjective night (Nikaido and Takahashi, 1998). A similar rhythm in cAMP content is also observed in chick retinal photoreceptors (Ivanova and Iuvone, 

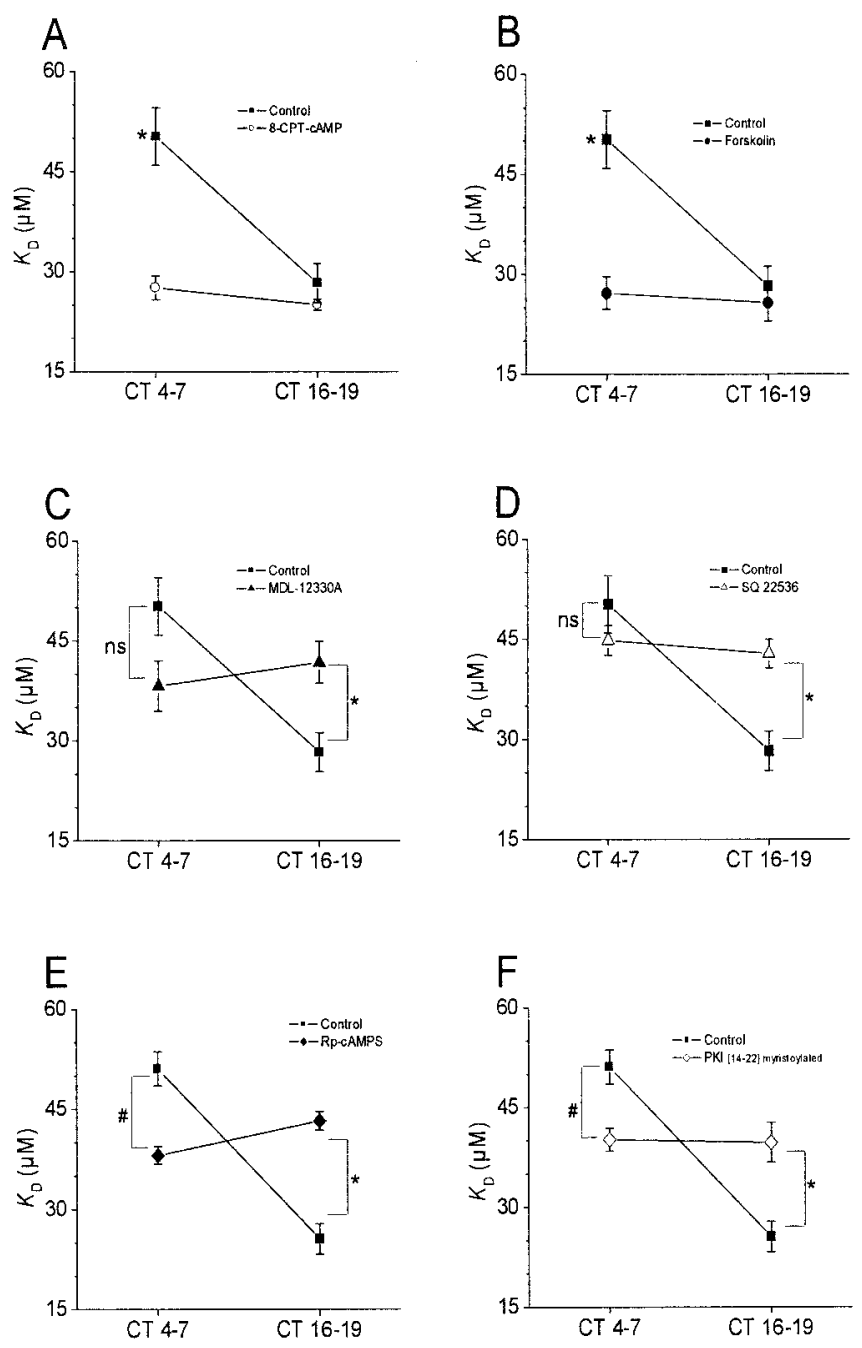

Figure 2. Phase-dependent modulation of CGMP-gated channels by cAMP. Experiments were performed on cones free-running on the second day of DD in vitro. Drugs were applied starting 1.5 -2 hr before inside-out patch recordings were made at CT4 -7 and CT16 - 19. A, The membrane-permeable cAMP analog 8-CPT-CAMP $(500 \mu \mathrm{M})$ caused a significant decrease in the mean $K_{D}$ of CGMP-gated channels during the subjective day (CT4 -7) but not during the subjective night (CT16 - 19). B, A similar effect was produced by forskolin (20 $\mu \mathrm{M})$. C, D, The adenylate cyclase inhibitors MDL-12330A (50 $\mu \mathrm{M}$ ) and SQ $22536(75 \mu \mathrm{M})$ significantly increased the $K_{D}$ of CGMP-gated channels during the subjective night but not during the subjective day. This was also seen with the PKA inhibitors Rp-CAMPS (50 $\mu \mathrm{m})$ and myristoyl-PKI ${ }_{[14-22]}(10 \mu \mathrm{M})(E, F)$. The symbols * and \# indicate $p<0.05$.

2003), which suggested the possibility that cAMP is part of the circadian output pathway that leads to modulation of CNGCs. To test this hypothesis, we examined the effects of agents that enhance or inhibit cAMP signaling cascades on the gating properties of cone CNGCs at different times of day. In these experiments, drugs were applied to intact cells $2 \mathrm{hr}$ before inside-out patch recordings were made from cones on the second day of DD. Exposing intact cells to $500 \mu \mathrm{M}$ 8-CPT-cAMP, a membranepermeable cAMP analog, caused a significant decrease in the apparent $K_{\mathrm{D}}$ of CNGCs in patches excised during the subjective day, when intracellular cAMP levels are normally at their nadir. However, 8CPT-cAMP had no effect on channel $K_{\mathrm{D}}$ during the subjective night (Fig. $2 \mathrm{~A}$ ). The same pattern was observed in intact cells treated for $2 \mathrm{hr}$ with $20 \mu \mathrm{M}$ forskolin, an activator of adenylate cyclase (Fig. $2 \mathrm{~B}$ ). Thus, exogenously applied cAMP protagonists evoke phase-dependent modulation of cone CNGCs.
We used two different pharmacological approaches to test a role for endogenous cAMP in circadian regulation of CNGCs. First, we examined the actions of the adenylate cyclase inhibitors MDL-12330A (50 $\mu \mathrm{M})$ or SQ-22536 $(75 \mu \mathrm{M})$, which should dampen or eliminate rhythms in intracellular cAMP levels. Exposure of intact photoreceptors to these agents for $2 \mathrm{hr}$ caused an increase in the apparent $K_{\mathrm{D}}$ of CNGCs in patches excised during the subjective night, when cAMP is normally at its peak, as well as a decrease in $K_{\mathrm{D}}$ during the subjective day, when cAMP levels are lower (Fig. 2C,D). A second approach is based on the fact that many of the actions of cAMP are mediated by PKA. We observed that two structurally and mechanistically distinct PKA inhibitors, Rp-cAMP-S or myristoyl-PKI ${ }_{[14-22]}$, eliminated circadian modulation of CNGCs by causing an increase in the apparent $K_{\mathrm{D}}$ during the subjective night as well as a small decrease in the $K_{\mathrm{D}}$ of CNGCs during the subjective day (Fig. $2 E, F$ ). Indeed, the pattern observed with these two structurally and mechanistically distinct PKA inhibitors is similar to that observed with inhibitors of adenylate cyclase. These data provide a second line of support for the hypothesis that endogenous cAMP is a component of the circadian output pathway leading to modulation of the affinity of cone CNGCs for their activating ligand. These data further suggest that cAMP acts at least in part through PKA to modulate CNGCs of chick cone photoreceptors.

\section{Role of Erk in cAMP modulation of cone CNGCs}

We have previously shown that circadian control of cone cGMPgated channels requires MEK-dependent activation of the MAP kinase Erk (Ko et al., 2001). Therefore, we investigated whether Erk is a component of the signaling cascade that leads to phasedependent modulation of CNGCs by cAMP. We have previously shown that treatment with the MEK1 inhibitor PD98059 (50 $\mu \mathrm{M})$ increases the $K_{\mathrm{D}}$ of cGMP-gated channels during the subjective night but has no effect during the subjective day. In the present study, we observed that a second MEK1 inhibitor, U0126 (30 $\mu \mathrm{M}$ ), produced similar effects on channel gating (Fig. 3C). Moreover, the effects of cAMP protagonists on CNGCs during the subjective day were reduced by either PD98059 or U0126 (Fig. 3). This observation is consistent with the theory that cAMP acts at least in part through Erk to modulate CNGCs. However, it seems likely that cAMP can activate additional pathways. Thus, PD98059 and U0126 were not able to convert nighttime behavior to daytime behavior in the presence of cAMP protagonists. This result was unexpected, and it suggests that the actions of cAMP on CNGCs are not entirely mediated through Erk signaling cascades, at least not in the presence of high intracellular cAMP activity.

Direct biochemical evidence also suggested an interaction between CAMP and Erk signaling in cones. Thus, we observed that application of 8-CPT-cAMP $(500 \mu \mathrm{M})$ or forskolin $(20 \mu \mathrm{M})$ evoked an increase in Erk phosphorylation. As with our previous study (Ko et al., 2001), we observed that basal Erk diphosphorylation was greater during the subjective night (CT17) than during the subjective day (CT5) in control cells (Fig. 4A). Application of either 8-CPT-cAMP or forskolin for $2 \mathrm{hr}$ increased Erk diphosphorylation, especially during the subjective day (measured at CT5), and also during subjective night (measured at CT17) (Fig. $4 A$ ). The effects of forskolin on Erk diphosphorylation were rapid in onset, and could be observed after a 15 min exposure (Fig. $4 \mathrm{~B}$ ). It bears noting that a $15 \mathrm{~min}$ exposure to forskolin is insufficient to cause modulation of CNGCs, whereas $2 \mathrm{hr}$ is sufficient (Fig. 4C). In a converse set of experiments, we observed that $2 \mathrm{hr}$ treatment with the adenylate cyclase inhibitor MDL-12330A 

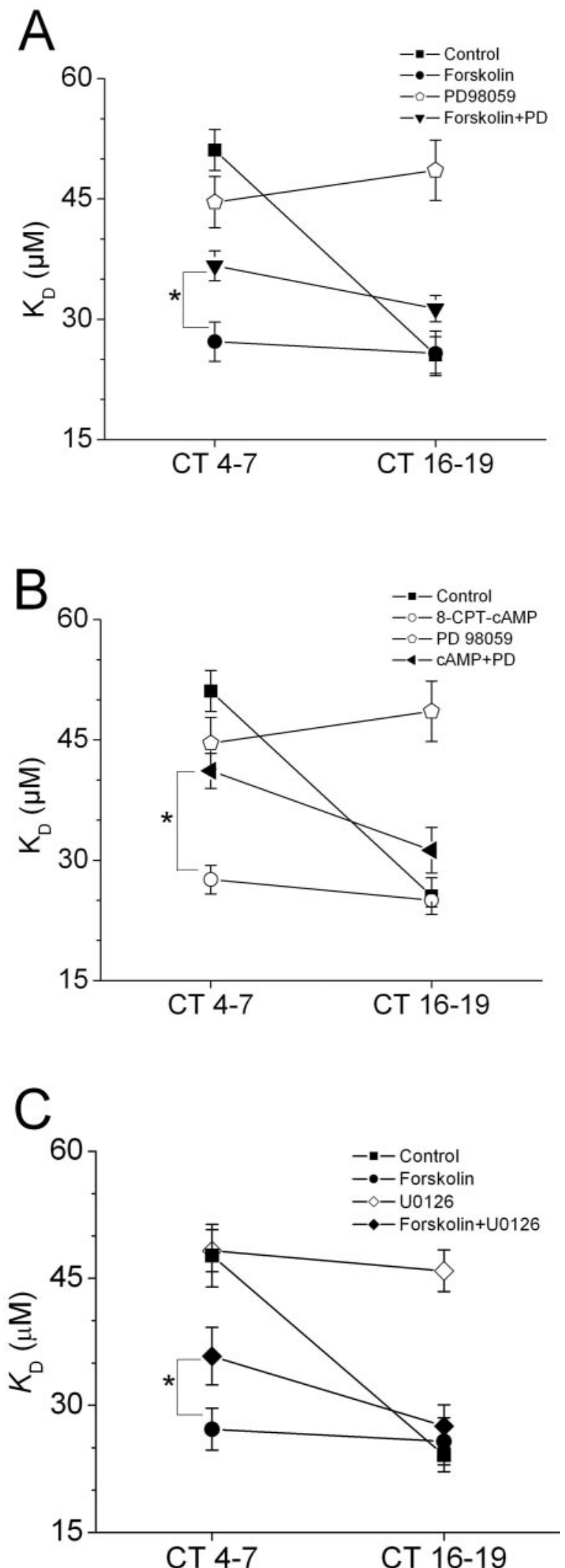

Figure 3. Erk mediates some of the effects of CAMP on CGMP-gated channels. A, Treatment with the selective MEK1 inhibitor PD98059 $(50 \mu \mathrm{M})$ increases the mean $K_{D}$ of CGMP-gated channels during the subjective night (CT 16-19) but has no effect during the subjective day (CT $4-7)$. B, The effects of forskolin $(20 \mu \mathrm{M})$ or 8-CPT-CAMP $(500 \mu \mathrm{m})$ on cGMP-gated channels during the subjective day (CT 4-7) were reduced by PD98059. C, The MEK1/2 inhibitor U0126 $(30 \mu \mathrm{M})$ produced effects similar to PD98059. ${ }^{*} p<0.05$.
A
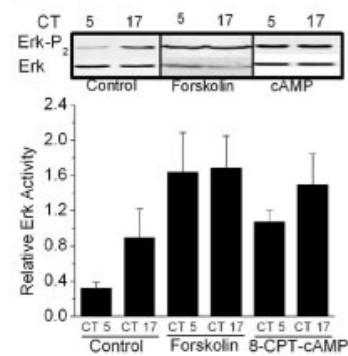

B

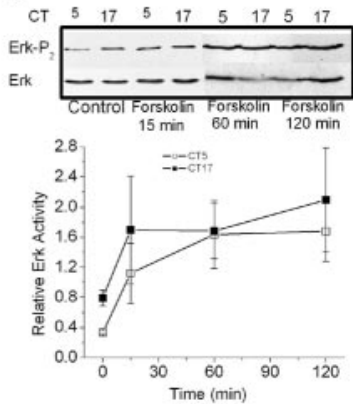

C

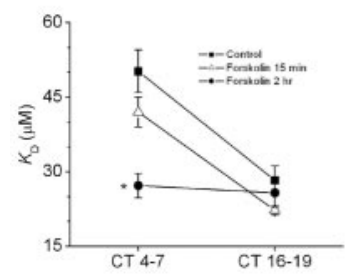

D
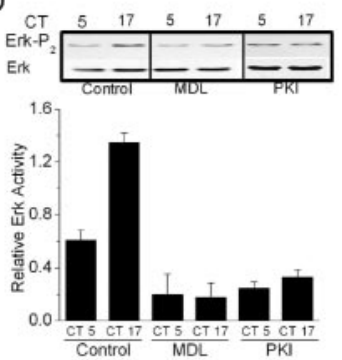

Figure 4. Erk activation is driven by CAMP in chick cones. A, Erk diphosphorylation is substantially greater during the subjective night (CT17) than during the subjective day (CT5) in control cells. Cells treated for $2 \mathrm{hr}$ with forskolin $(20 \mu \mathrm{M})$ or 8-CPT-CAMP (500 $\mu \mathrm{M})$ exhibit enhanced Erk diphosphorylation during both the subjective night and day. $B$, Treatment with forskolin for $15 \mathrm{~min}, 60 \mathrm{~min}$, or $2 \mathrm{hr}$ increases Erk activity during both the subjective day and night. C, However, treatment with forskolin for 15 min does not change the apparent affinities of CNGCs for activating ligand during either the subjective day and night, whereas a $2 \mathrm{hr}$ treatment is effective. D, Treatment with MDL-12330A (MDL) $(50 \mu \mathrm{M})$ or myristoyl-PKI ${ }_{[14-22]}$ (PKI) $(10 \mu \mathrm{m})$ for $2 \mathrm{hr}$ significantly decreased Erk diphosphorylation during both the subjective day and night. In this figure, representative blots are shown above results of densitometric analysis $(n=4)$. Ordinates of the graphs represent signal for diphosphorylated Erk divided by total Erk. ${ }^{*} p<0.05$ compared with all other data points.

$(50 \mu \mathrm{M})$ or the selective PKA inhibitor myristoyl-PKI $\mathrm{I}_{[14-22]}(10 \mu \mathrm{M})$ decreased Erk diphosphorylation during both the subjective day and subjective night (Fig. 4D). Because it is the diphosphorylated form of Erk that is active, these data indicate that in chick retina, adenylate cyclase, and endogenous intracellular cAMP regulate Erk activation during the circadian cycle via a PKA-dependent pathway. This part of the circadian output pathway can occur quickly, and the ratelimiting steps appear to lie in the cascades that intervene between Erk and the CNGCs.

\section{Ras and B-Raf are required for circadian and cAMP modulation of CNGCs}

In the photoreceptive chick pineal gland, activation of the small GTPase Ras is under circadian control (Hayashi et al., 2001). In chick retina, we have used a pull-down assay to show that activation of the small GTPase Ras is also under circadian control, with peak activity occurring during the subjective night (Fig. $5 \mathrm{~A}$ ). Is Ras is part of the pathway leading from cAMP to Erk activation? We used two different approaches to test this hypothesis. The first approach is based on the fact that Ras activation requires exchange of GTP for GDP, typically catalyzed by guanine nucleotide exchange proteins, and also modification by a protein farnesyl transferase necessary for membrane recruitment. The latter process is amenable to pharmacological perturbation. We observed that treatment of photoreceptors with the farnesyl transferase inhibitor manumycin A (1 $\mu \mathrm{M})$ for $2 \mathrm{hr}$ before patch excision had no effect on cGMP-gated channels in patches excised during the subjective day, but signifi- 


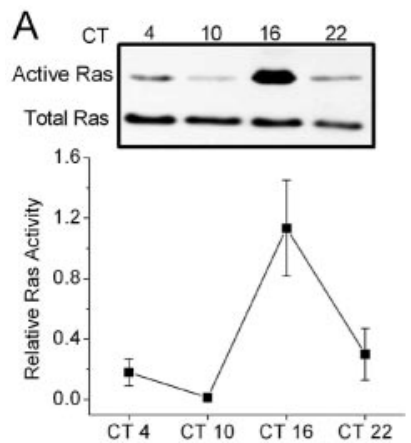

B
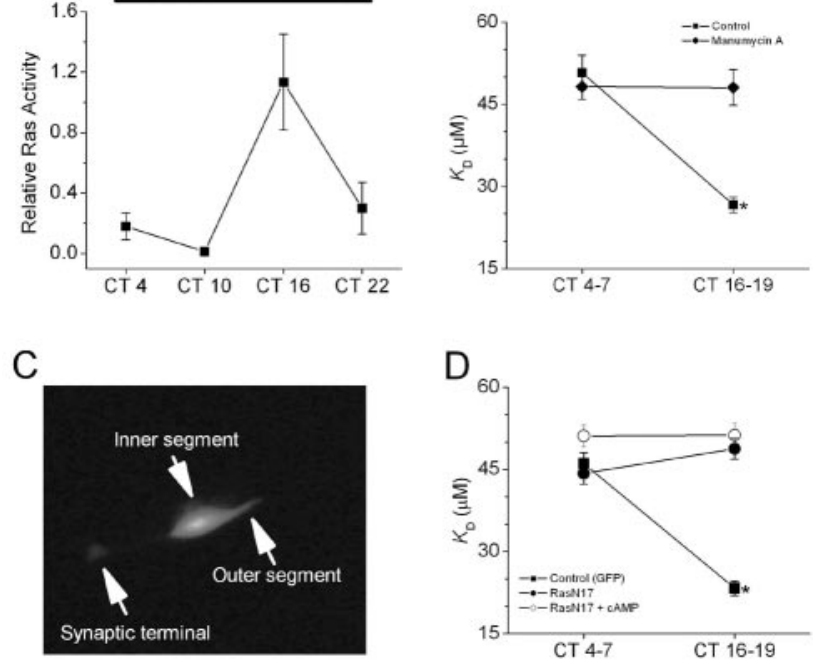

Figure 5. Ras activation is under circadian control and is required for CAMP modulation of cGMP-gated channels. A, Ras activation measured by a pull-down assay in chick retinal cells at various times on the second day of DD. Ras activation peaked during the middle of the subjective night $(n=3)$. B, Application of the farnesyl transferase inhibitor manumycin A (1 $\mu \mathrm{m})$ for $2 \mathrm{hr}$ significantly increased the mean $K_{D}$ for activation of CNGCs during the subjective night (CT16 19) but not the subjective day (CT4-7). C, Example of GFP expression in a chick cone photoreceptor after biolistic transfection. D, Control cells (expressing GFP alone, filled squares) displayed a normal circadian modulation of cGMP-gated channels. Cells cotransfected with GFP and RasN17 showed no circadian rhythm owing to an increased $K_{D}$ during the subjective night (filled circles). Cells expressing GFP and Ras N17 (open circles) did not show a decrease in $K_{D}$ after a 2 hr exposure to $500 \mu \mathrm{m} 8$-CPT-CAMP. ${ }^{*} p<0.05$ compared to all other data points.

cantly increased the apparent $K_{\mathrm{D}}$ during the subjective night, when Ras is normally most active (Fig. $5 B$ ).

Although this observation supports the hypothesis, it bears noting that monomeric GTPases of the Ras family are not the only proteins that require farnesylation for normal biological activity. Therefore, we used another approach to test the role of Ras specifically, namely overexpression of a selective dominantnegative mutant, the S17N mutation of Ras (RasN17). Using biolistic procedures, we transfected cultured cones on the first day of DD with a combination of vectors encoding GFP and RasN17 (both under the control of a CMV promoter) or with GFP alone. The transfections were not performed until after the cells were entrained to LD 12:12 for $5 \mathrm{~d}$, which ensured that inhibition of Ras signaling did not disrupt entrainment of the cells. Transfected cells could be readily observed at the time of recording using fluorescence optics (Fig. 5C). On the second day of $\mathrm{DD}$, inside-out patches were excised from cones during the subjective day (CT4-7) or the subjective night (CT16-19). Control cells (expressing GFP alone) displayed a normal circadian modulation of CNGCs, with a high apparent $K_{\mathrm{D}}$ during the subjective day and a reduced $K_{\mathrm{D}}$ during the subjective night. By contrast, cells cotransfected with GFP and RasN17 showed no circadian rhythm owing to an increased $K_{\mathrm{D}}$ during the subjective night (Fig. 5D). Together, these data indicate that Ras activation is necessary for the circadian rhythm in the affinity of CNGCs for activating ligand in chick cones. Moreover, Ras activation is required for cAMP modulation of cone CNGCs. Thus, $500 \mu \mathrm{M}$ 8-CPT-cAMP had no effect on the $K_{\mathrm{D}}$ of CNGCs in cells expressing GFP and RasN17 (Fig. 5D). This result indicates that Ras is
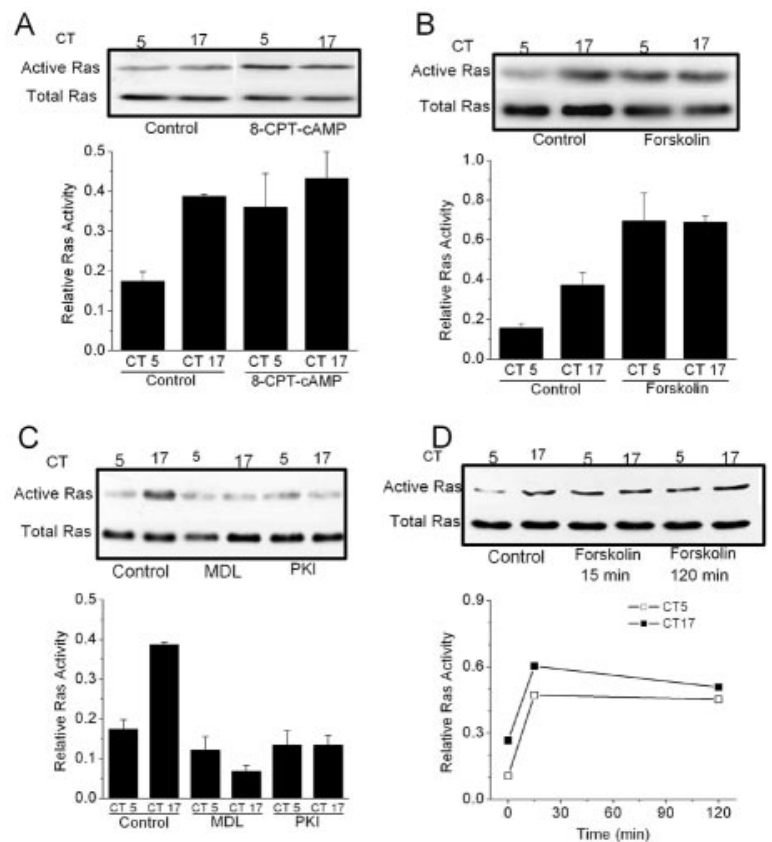

Figure 6. An endogenous CAMP-dependent signaling pathway modulates Ras activation in chick cones. Cells were treated with drugs for $2 \mathrm{hr}$ starting at CT3 or CT15 on the second day of DD. Ras activation was measured at CT5 or CT17. A, B, Ras activation is greater during the subjective night (CT17) than during the subjective day (CT5) in control cells. Cells treated with 8-CPT-CAMP $(500 \mu \mathrm{m})$ or forskolin $(20 \mu \mathrm{m})$ exhibit elevated Ras activity during both the subjective night (CT17) and subjective day (CT5). C, Conversely, treatment with the adenylate cyclase inhibitor MDL-12330A (50 $\mu \mathrm{m})$ or the PKA inhibitor myristoyl-PKI ${ }_{[14-22]}(10 \mu \mathrm{m})$ decreased Ras activity during both the subjective day and night. $D$, Treatment with forskolin for 15 min causes Ras activation during both the subjective day and night. In this figure, results of representative assays are shown above results of densitometric analysis $(n=4)$.

necessary for the circadian phase-dependent modulation of cone cGMP-gated channels by cAMP.

Consistent with this finding, we have also observed that cAMP protagonists can evoke Ras activation in cones. Thus, a $2 \mathrm{hr}$ exposure to 8-CPT-cAMP causes an increase of Ras activation during the subjective day (Fig. 6A). Forskolin also evoked Ras activation (Fig. 6B), and this effect was inhibited by myristoyl$\mathrm{PKI}_{[14-22]}$ (data not shown). Conversely, treatment with the adenylate cyclase inhibitor MDL-12330A (50 $\mu \mathrm{M})$ or the PKA inhibitor myristoyl-PKI ${ }_{[14-22]}(10 \mu \mathrm{M})$ decreased Ras activation during both the subjective day and subjective night (Fig. 6C). As with Erk, forskolin-evoked activation of Ras is rapid compared with its effects on CNGCs, because robust Ras activation occurred within $15 \mathrm{~min}$ (Fig. 6D).

In neuronal systems, cAMP can stimulate pathways leading to activation of another small GTPase known as Rap1, which in turn can lead to Erk activation (de Rooij et al., 1998; Kawasaki et al., 1998; Grewal et al., 1999, 2000). However, we have been unable to detect Rap1 in cultured chick photoreceptors by immunoblot analysis using a polyclonal antibody against Rap1 that can readily detect Rap1 expression in chick brain at the same developmental stage. Moreover, cotransfection with GFP and a Rap dominantnegative mutant RapN17 (Schmitt and Stork, 2000) had no effect on circadian or CAMP modulation of CNGCs (data not shown).

In signaling systems studied to date, the actions of Ras are mediated by MEK kinases of the Raf family, including Raf-1 (Peyssonnaux et al., 2000; Peyssonnaux and Eychene, 2001), and B-Raf (Kao et al., 2001). We have investigated whether circadian and cAMP modulation of CNGCs is mediated by members of this 
A

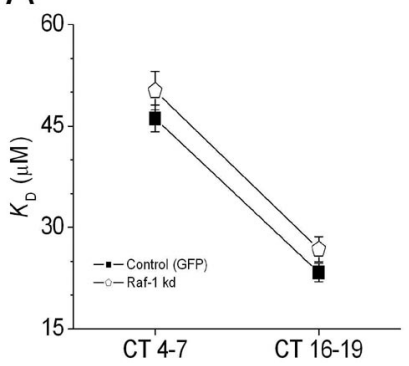

B

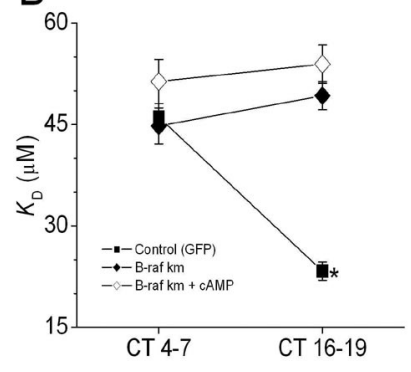

Figure 7. Circadian and CAMP modulation of CGMP-gated channels requires B-Raf, but Raf-1 is not necessary. $A$, Cones cotransfected with GFP and a Raf-1 dominant negative mutant (Raf-1 kd) exhibit normal circadian regulation of CGMP-gated channels (open pentagon). $B$, Cotransfection with GFP and the B-Raf dominant-negative mutant (B-Raf km) eliminated the circadian rhythm in CGMP-gated channel properties owing to an increased $K_{D}$ during the subjective night (filled diamonds). The B-Raf dominant-negative construct also blocked responses to $500 \mu \mathrm{m}$ 8CPT-cAMP (open diamonds). ${ }^{*} p<0.05$ compared to all other data points.

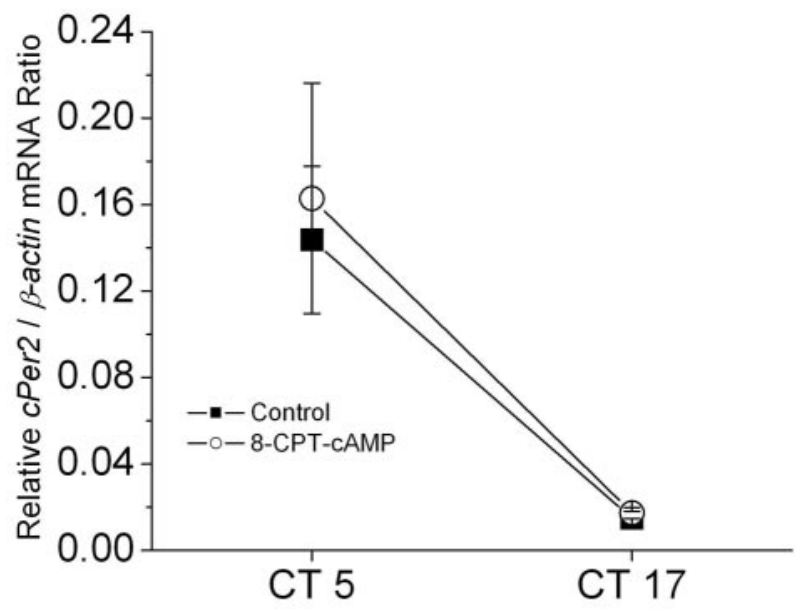

Figure 8. Exposure to a cAMP analog for $2 \mathrm{hr}$ does not affect transcription of a core clock component in embryonic chick retina. Cultured cone photoreceptors were treated with $500 \mu \mathrm{M}$ 8-CPT-CAMP or vehicle for $2 \mathrm{hr}$ starting at $\mathrm{CT} 3$ or $\mathrm{CT} 15$ on the second day of DD and $\mathrm{CPer} 2 \mathrm{mRNA}$ was measured by real-time quantitative RT-PCR at $C T 5$ and $C T$ 17. This treatment did not affect cPer2 levels at either time of day in cultured chick photoreceptors.

family of proteins. We observed that cotransfection with GFP and a Raf-1 dominant-negative mutant (Raf-1-kd) had no effect on circadian modulation of CNGCs (Fig. 7A). However, cotransfection with GFP and a B-Raf dominant-negative mutant (B-Raf$\mathrm{km}$ ) blocked the circadian modulation of CNGCs, as well as the effects of 8-CPT-cAMP on these channels (Fig. 7B). These two Raf family dominant-negative constructs are in the same expression vector and driven by the same promoter. These data are consistent with the theory that a pathway comprised of Ras, B-Raf, MEK, and Erk is essential for circadian modulation of photoreceptor CNGCs.

The effects of $2 \mathrm{hr}$ treatments with cAMP protagonists on Erk, Ras, and CNGCs are not associated with changes in the dynamic state of the circadian oscillator (Fig. 8). Stated in another way, cAMP is functioning as a true clock output, and the effects of cAMP pathway protagonists and antagonists noted above are not a simple consequence of clock phase shifting. If they were producing a significant effect on the overall dynamic state of the circadian oscillator, than one would expect to see a change in the levels of one of the cycling clock gene transcripts. To test this, we

measured levels of transcripts encoded by the clock gene $c P e r 2$ in cultured photoreceptors by means of real-time quantitative RTPCR. This transcript is rhythmically expressed in avian circadian oscillator systems (Doi et al., 2001) and can therefore serve as a read-out of the dynamic state of the clock. We have previously shown that cPer2 mRNA levels in retina on the second day of DD are highest during the subjective day (Ko et al., 2003). Exposure to 8-CPT-cAMP $(500 \mu \mathrm{M})$ for $2 \mathrm{hr}$ starting at CT3 or CT15 had no effect on $c$ Per 2 transcript levels at either circadian phase (Fig. 8). Note that this does not exclude that cAMP can phase-shift the retinal oscillator, indeed it is known to do so in Xenopus retinal photoreceptors (Hasegawa and Cahill, 1998). However, the consequences of clock phase-shifting would normally be observed on subsequent cycles, rather than immediately after cessation of a 2 hr drug treatment.

\section{Discussion}

We have previously shown that the CNGCs of chicken cone photoreceptors are under circadian control such that they have a higher affinity for activating ligand during the subjective night (Ko et al., 2001, 2003). Computational (Ko et al., 2001; Pugh et al., 1999) and experimental (Savchenko et al., 2001) evidence suggests that this type of modulation should markedly affect the dynamics of phototransduction. In the present study, we have demonstrated that photoreceptor circadian oscillators modulate cone CNGCs by a pathway that uses cAMP, Ras, and Erk. There are circadian rhythms in the cAMP content of retinal photoreceptors (Ivanova and Iuvone, 2003), and this messenger appears to drive Ras activation through a pathway that depends on protein kinase A. Activated Ras in turn evokes Erk activation by a pathway that requires B-Raf and MEK.

One of the key observations of this study is that Ras activation is under circadian control in vertebrate photoreceptors, and that Ras activation is essential for circadian control of cone CNGCs. Ras and related small GTPases play a central position in mediating many cellular processes, and there are a number of demonstrated and putative downstream effectors of Ras (Vojtek and Der, 1998; Takai et al., 2001). Therefore, it is possible that Ras controls circadian output pathways other than the one demonstrated here. For example, Ras activation in postmitotic cells, acting through effector systems such as Erk, Ral, and PI3K, can lead to changes in gene expression and remodeling of the cell cytoskeleton. Both of these processes could play a role in modulating overall circadian control of photoreceptors, because many vertebrate photoreceptors, including those of birds, undergo extensive retinomotor movements in response to changes in ambient illumination and circadian control processes (Drenckhahn and Wagner, 1985; Burnside, 2001). Retinomotor movements require extensive remodeling of the cytoskeleton, and it would interesting to know if Ras plays a role in regulating this process.

The cAMP content of pineal and retinal photoreceptors is maximal during the subjective night (Nikaido and Takahashi, 1998; Ivanova and Iuvone, 2003), and at least in retina this rhythm appears to drive a rhythm in melatonin synthesis and secretion (Ivanova and Iuvone, 2003). Our data provide several independent lines of evidence that endogenous cAMP is also a component of the circadian output pathway that leads to Erk activation and modulation of CNGCs. Specifically, two different inhibitors of adenylate cyclase and two different inhibitors of PKA eliminate circadian modulation of CNGCs, which lock into a low-affinity state that approaches that of the normal subjective day. Conversely, pharmacological activation of adenylate cyclase (by forskolin), or application of a membrane-permeable cAMP 
analog, causes CNGCs to lock into a high-affinity state characteristic of the subjective night. These treatments also produce corresponding circadian phase-dependent effects on Ras and Erk activation, as cAMP protagonists activate these enzymes, whereas adenylate cyclase inhibitors eliminate the normal nocturnal increase in their activity. However, certain details of our results suggest that cAMP can activate multiple cascades that eventually lead to modulation of CNGCs, at least under certain conditions. For example, CNGCs continued to exhibit a relatively high affinity for cGMP in the combined presence of forskolin and MEK inhibitors. It is possible that additional pathways become available to cAMP once MEK is inhibited, possibly because of diversion of active Ras toward other effectors. Alternatively, it is possible that forskolin during the nighttime produces very high and nonphysiological intracellular cAMP levels, leading to activation of pathways that would not otherwise occur in these cells. In any case, it is clear that Ras and Erk are essential components of the normal circadian output pathway driven by endogenous cAMP, and it seems equally clear that Ras and Erk are not the only pathways driven by cAMP.

Many intracellular proteins are substrates for PKA, and there are other CAMP-binding proteins that can transduce the actions of this second messenger (Richards, 2001; Enserink et al., 2002). As a result, cAMP has highly pleiotropic actions in cells and appears to be a component of multiple circadian output pathways in vertebrate retinal photoreceptors. As noted above, cAMP plays a role in circadian clock regulation of melatonin secretion from retinal photoreceptors (Ivanova and Iuvone, 2003). Interestingly, this does not appear to be the case in the chick pineal gland (Zatz, 1992). Similarly, there is evidence that cAMP can stimulate retinomotor movements (Besharse et al., 1982; Burnside et al., 1982; Burnside and Ackland, 1984; Burnside, 2001), but it is not known whether cAMP drives the circadian rhythm in this process. It should also be noted that cAMP can reset the circadian oscillators of Xenopus retinal photoreceptors (Hasegawa and Cahill, 1998). Clock resetting caused by altered cAMP signaling cannot explain our results, because the treatment durations that we used are short compared with a circadian cycle, and we observed robust effects of cAMP protagonists on CNGCs, Ras, and Erk activities without altering $c$ Per 2 mRNA levels.

In neurons, there is precedent for cAMP causing activation of Ras/Raf-1/Erk (Ambrosini et al., 2000; Iida et al., 2001) as well as Rap-1/B-Raf/Erk signal pathways (Vossler et al., 1997; Dugan et al., 1999). Our data infer that a Ras/B-Raf/Erk pathway controls the circadian modulation of CNGCs and is a downstream effector of cAMP signaling. This pathway for Erk activation is somewhat unusual, but a similar pathway has been identified in melanocytes and melanoma cells based on similar lines of evidence (Busca et al., 2000). Thus, signaling in melanocytes and cone photoreceptors is blocked by dominant-negative forms of Ras and B-Raf, and resistant to dominant-negative forms of Rap1 and Raf-1 (indeed Rap1 cannot be detected in chick cone photoreceptors). Moreover, it has been known for some time that activated Ras can bind to and activate B-Raf in vitro (Jaiswal et al., 1994; Ohtsuka et al., 1996; Marais et al., 1997).

The mechanism by which cAMP causes activation of Ras in photoreceptors is unknown, but there are several possibilities based on precedents in other cells. However, one class of these possibilities can be excluded. Thus, activation of Erk and modulation of CNGCs in photoreceptors is blocked by two structurally dissimilar PKA inhibitors, which suggests that the effects observed here are not caused by any of the guanine nucleotide exchange factors that bind cAMP directly (Richards, 2001; Enserink et al., 2002). This still leaves many other possibilities. For example, PKA can cause activation of Src family kinases that feed into Ras pathways in some neuronal and fibroblast cell lines (Klinger et al., 2002). Moreover, a number of molecules that modulate Ras family proteins are substrates for PKA (Baouz et al., 2001). These include a GTPase activating protein known as neurofibromin, the product of the neurofibromatosis-1 gene (Ballester et al., 1990; Martin et al., 1990; Xu et al., 1990; Izawa et al., 1996; Tokuo et al., 2001). Interestingly, the Drosophila ortholog of this gene plays a role in circadian output pathways, because mutations at this locus lead to a loss of locomotor activity rhythms and abnormally elevated Erk activity but have no effect on the core oscillator (Williams et al., 2001).

The mechanism whereby the core oscillator drives a circadian rhythm in cAMP content of cone photoreceptors is not well understood. We observed that adenylate cyclase activity is required for circadian rhythms in Ras, Erk, and CNGCs, and it is certainly possible that the activity or expression of this enzyme is under circadian control in cones. In fact, circadian rhythms in adenylate cyclase activity are observed in chick pineal photoreceptors (Nikaido and Takahashi, 1998). However, this is not the only plausible explanation for the present results. It is also possible that a cAMP phosphodiesterase (PDE) is under circadian control. Note that a rhythm in PDE activity would be ineffective in the absence of substrate to work on, so that in either case, inhibition of adenylate cyclase would be expected to produce the effects that we observe.

The present results provide information on the kinetics and dynamics of the circadian output pathway that leads to modulation of CNGCs. We observed that forskolin could lead to robust activation of Erk within 15 min or less. However, a 15 min exposure to forskolin was not sufficient to cause modulation of CNGCs, which instead requires at least $1 \mathrm{hr}$. In other words, the cascades leading to Erk are relatively fast, whereas the pathway from Erk to the channels is slow. It is possible that the pathway from Erk to CNGCs entails a large number of individual steps, but it is also possible that a small number of steps occur with a low probability, thereby leading to a delay in channel modulation. Alternatively, Erk may have to diffuse a considerable distance from its site of activation to make physical contact with membranes containing CNGCs and associated proteins. Our data on cPer2 indicate that this delay is not a consequence of clock phaseshifting. It also bears noting that we observed that forskolin consistently evoked stimulation of Erk diphosphorylation during the day and also during the night. Conversely, adenylate cyclase inhibitors caused inhibition of Erk, especially during the night, but also during the day. In other words, the circadian rhythm of Erk activation uses only a portion of the dynamic range that is available for this signaling system. Nevertheless, this relatively low amplitude rhythm is sufficient to account for circadian modulation of CNGCs, and pushing Erk activation outside of this range does not produce additional effects on channel gating. This observation is relevant to our previous study of dopaminergic control of CNGCs in cone photoreceptors (Ko et al., 2003), because that neurotransmitter produced activation of Erk during the subjective day, but inhibition of Erk during the subjective night. In other words, the circadian oscillator does not saturate the Erk cascade during the subjective night, and that cannot explain why dopamine fails to stimulate Erk at that phase of the circadian cycle.

In summary, we have demonstrated that at least a portion of the circadian output pathway leading to modulation of cone CNGCs in photoreceptors requires cAMP, PKA, Ras, and an iso- 
form of Raf, leading to a relatively rapid activation of Erk. That MAP kinase in turn leads to a slower modulation of the channels that, based on kinetics, may be the result of a fairly extended cascade.

\section{References}

Adler R, Hatlee M (1989) Plasticity and differentiation of embryonic retinal cells after terminal mitosis. Science 243:391-393.

Adler R, Lindsey JD, Elsner CL (1984) Expression of cone-like properties by chick embryo neural retina cells in glial-free monolayer cultures. J Cell Biol 99:1173-1178.

Ambrosini A, Tininini S, Barassi A, Racagni G, Sturani E, Zippel R (2000) cAMP cascade leads to Ras activation in cortical neurons. Brain Res Mol Brain Res 75:54-60.

Ballester R, Marchuk D, Boguski M, Saulino A, Letcher R, Wigler M, Collins F (1990) The NF1 locus encodes a protein functionally related to mammalian GAP and yeast IRA proteins. Cell 63:851-859.

Baouz S, Jacquet E, Accorsi K, Hountondji C, Balestrini M, Zippel R, Sturani E, Parmeggiani A (2001) Sites of phosphorylation by protein kinase A in CDC25Mm/GRF1, a guanine nucleotide exchange factor for Ras. J Biol Chem 276:1742-1749.

Bassi CJ, Powers MK (1987) Circadian rhythm in goldfish visual sensitivity. Invest Ophthalmol Vis Sci 28:1811-1815.

Belecky-Adams T, Cook B, Adler R (1996) Correlations between terminal mitosis and differentiated fate of retinal precursor cells in vivo and in vitro: analysis with the "window-labeling" technique. Dev Biol 178:304-315.

Besharse JC, Dunis DA, Burnside B (1982) Effects of cyclic adenosine 3', $5^{\prime}$-monophosphate on photoreceptor disc shedding and retinomotor movement. Inhibition of rod shedding and stimulation of cone elongation. J Gen Physiol 79:775-790.

Burns ME, Baylor DA (2001) Activation, deactivation, and adaptation in vertebrate photoreceptor cells. Annu Rev Neurosci 24:779-805.

Burnside B (2001) Light and circadian regulation of retinomotor movement. Prog Brain Res 131:477-485.

Burnside B, Ackland N (1984) Effects of circadian rhythm and cAMP on retinomotor movements in the green sunfish, Lepomis cyanellus. Invest Ophthalmol Vis Sci 25:539-545.

Burnside B, Evans M, Fletcher RT, Chader GJ (1982) Induction of darkadaptive retinomotor movement (cell elongation) in teleost retinal cones by cyclic adenosine 3',5-monophosphate. J Gen Physiol 79:759-774.

Busca R, Abbe P, Mantoux F, Aberdam E, Peyssonnaux C, Eychene A, Ortonne JP, Ballotti R (2000) Ras mediates the cAMP-dependent activation of extracellular signal-regulated kinases (ERKs) in melanocytes. EMBO J 19:2900-2910.

Cahill GM, Besharse JC (1995) Circadian rhythmicity in vertebrate retinas: regulation by a photoreceptor oscillator. Prog Retin Eye Res 14:267-291.

de Rooij J, Zwartkruis FJ, Verheijen MH, Cool RH, Nijman SM, Wittinghofer A, Bos JL (1998) Epac is a Rap1 guanine-nucleotide-exchange factor directly activated by cyclic AMP. Nature 396:474-477.

Dearry A, Barlow RB Jr (1987) Circadian rhythms in the green sunfish retina. J Gen Physiol 89:745-770.

Doi M, Nakajima Y, Okano T, Fukada Y (2001) Light-induced phase-delay of the chicken pineal circadian clock is associated with the induction of cE4bp4, a potential transcriptional repressor of cPer2 gene. Proc Natl Acad Sci USA 98:8089-8094.

Drenckhahn D, Wagner HJ (1985) Relation of retinomotor responses and contractile proteins in vertebrate retinas. Eur J Cell Biol 37:156-168.

Dugan LL, Kim JS, Zhang Y, Bart RD, Sun Y, Holtzman DM, Gutmann DH (1999) Differential effects of cAMP in neurons and astrocytes. Role of B-raf. J Biol Chem 274:25842-25848.

Enserink JM, Christensen AE, de Rooij J, van Triest M, Schwede F, Genieser HG, Doskeland SO, Blank JL, Bos JL (2002) A novel Epac-specific cAMP analogue demonstrates independent regulation of Rap1 and ERK. Nat Cell Biol 4:901-906.

Grewal SS, York RD, Stork PJ (1999) Extracellular-signal-regulated kinase signaling in neurons. Curr Opin Neurobiol 9:544-553.

Grewal SS, Fass DM, Yao H, Ellig CL, Goodman RH, Stork PJ (2000) Calcium and cAMP signals differentially regulate cAMP-responsive elementbinding protein function via a Rap1-extracellular signal-regulated kinase pathway. J Biol Chem 275:34433-34441.
Hasegawa M, Cahill GM (1998) Cyclic AMP resets the circadian clock in cultured Xenopus retinal photoreceptor layers. J Neurochem 70:1523-1531.

Hayashi Y, Sanada K, Fukada Y (2001) Circadian and photic regulation of MAP kinase by Ras- and protein phosphatase-dependent pathways in the chick pineal gland. FEBS Lett 491:71-75.

Iida N, Namikawa K, Kiyama H, Ueno H, Nakamura S, Hattori S (2001) Requirement of Ras for the activation of mitogen-activated protein kinase by calcium influx, cAMP, and neurotrophin in hippocampal neurons. J Neurosci 21:6459-6466.

Ivanova TN, Iuvone PM (2003) Circadian rhythm and photic control of cAMP level in chick retinal cell cultures: a mechanism for coupling the circadian oscillator to the melatonin-synthesizing enzyme, arylalkylamine $N$-acetyltransferase, in photoreceptor cells. Brain Res 991:96-103.

Izawa I, Tamaki N, Saya H (1996) Phosphorylation of neurofibromatosis type 1 gene product (neurofibromin) by cAMP-dependent protein kinase. FEBS Lett 382:53-59.

Jaiswal RK, Moodie SA, Wolfman A, Landreth GE (1994) The mitogenactivated protein kinase cascade is activated by B-Raf in response to nerve growth factor through interaction with p21 ras. Mol Cell Biol 14:6944-6953.

Kao S, Jaiswal RK, Kolch W, Landreth GE (2001) Identification of the mechanisms regulating the differential activation of the MAPK cascade by epidermal growth factor and nerve growth factor in PC12 cells. J Biol Chem 276:18169-18177.

Kawasaki H, Springett GM, Mochizuki N, Toki S, Nakaya M, Matsuda M, Housman DE, Graybiel AM (1998) A family of cAMP-binding proteins that directly activate Rap1. Science 282:2275-2279.

Klinger M, Kudlacek O, Seidel MG, Freissmuth M, Sexl V (2002) MAP kinase stimulation by cAMP does not require RAP1 but SRC family kinases. J Biol Chem 277:32490-32497.

Ko GY, Ko ML, Dryer SE (2001) Circadian regulation of cGMP-gated cationic channels of chick retinal cones. Erk MAP Kinase and $\mathrm{Ca} 2+/$ calmodulin-dependent protein kinase II. Neuron 29:255-266.

Ko GY, Ko ML, Dryer SE (2003) Circadian phase-dependent modulation of cyclic GMP-gated channels of cone photoreceptors by dopamine and D2 agonist. J Neurosci 23:3145-3153.

Kramer RH, Molokanova E (2001) Modulation of cyclic-nucleotide-gated channels and regulation of vertebrate phototransduction. J Exp Biol 204:2921-2931

Li L, Dowling JE (1998) Zebrafish visual sensitivity is regulated by a circadian clock. Vis Neurosci 15:851-857.

Lu J, Zoran MJ, Cassone VM (1995) Daily and circadian variation in the electroretinogram of the domestic fowl: effects of melatonin. J Comp Physiol [A] 177:299-306.

Manglapus MK, Iuvone PM, Underwood H, Pierce ME, Barlow RB (1999) Dopamine mediates circadian rhythms of rod-cone dominance in the Japanese quail retina. J Neurosci 19:4132-4141.

Manglapus MK, Uchiyama H, Buelow NF, Barlow RB (1998) Circadian rhythms of rod-cone dominance in the Japanese quail retina. J Neurosci 18:4775-4784.

Marais R, Light Y, Paterson HF, Mason CS, Marshall CJ (1997) Differential regulation of Raf-1, A-Raf, and B-Raf by oncogenic ras and tyrosine kinases. J Biol Chem 272:4378-4383.

Martin GA, Viskochil D, Bollag G, McCabe PC, Crosier WJ, Haubruck H, Conroy L, Clark R, O'Connell P, Cawthon RM, et al. (1990) The GAPrelated domain of the neurofibromatosis type 1 gene product interacts with ras p21. Cell 63:843-849.

McGoogan JM, Cassone VM (1999) Circadian regulation of chick electroretinogram: effects of pinealectomy and exogenous melatonin. Am J Physiol 277:R1418-1427.

Molokanova E, Kramer RH (2001) Mechanism of inhibition of cyclic nucleotide-gated channel by protein tyrosine kinase probed with genistein. J Gen Physiol 117:219-234.

Nikaido SS, Takahashi JS (1998) Day/night differences in the stimulation of adenylate cyclase activity by calcium/calmodulin in chick pineal cell cultures: evidence for circadian regulation of cyclic AMP. J Biol Rhythms 13:479-493.

Ohtsuka T, Shimizu K, Yamamori B, Kuroda S, Takai Y (1996) Activation of brain B-Raf protein kinase by Rap1B small GTP-binding protein. J Biol Chem 271:1258-1261.

Peyssonnaux C, Eychene A (2001) The Raf/MEK/ERK pathway: new concepts of activation. Biol Cell 93:53-62.

Peyssonnaux C, Provot S, Felder-Schmittbuhl MP, Calothy G, Eychene A 
(2000) Induction of postmitotic neuroretina cell proliferation by distinct Ras downstream signaling pathways. Mol Cell Biol 20:7068-7079.

Pugh Jr EN, Nikonov S, Lamb TD (1999) Molecular mechanisms of vertebrate photoreceptor light adaptation. Curr Opin Neurobiol 9:410-418.

Rebrik TI, Korenbrot JI (1998) In intact cone photoreceptors, a $\mathrm{Ca}^{2+}$ dependent, diffusible factor modulates the cGMP-gated ion channels differently than in rods. J Gen Physiol 112:537-548.

Reme CE, Wirz-Justice A, Terman M (1991) The visual input stage of the mammalian circadian pacemaking system: I. Is there a clock in the mammalian eye? J Biol Rhythms 6:5-29.

Richards JS (2001) New signaling pathways for hormones and cyclic adenosine 3', 5'-monophosphate action in endocrine cells. Mol Endocrinol 15:209-218.

Rieke F, Schwartz EA (1994) A cGMP-gated current can control exocytosis at cone synapses. Neuron 13:863-873.

Savchenko A, Barnes S, Kramer RH (1997) Cyclic-nucleotide-gated channels mediate synaptic feedback by nitric oxide. Nature 390:694-698.

Savchenko A, Kraft TW, Molokanova E, Kramer RH (2001) Growth factors regulate phototransduction in retinal rods by modulating cyclic nucleotide-gated channels through dephosphorylation of a specific tyrosine residue. Proc Natl Acad Sci USA 98:5880-5885.

Schmitt JM, Stork PJ (2000) beta 2-adrenergic receptor activates extracellular signal-regulated kinases (ERKs) via the small G protein rap1 and the serine/threonine kinase B-Raf. J Biol Chem 275:25342-25350.

Shaw AP, Collazo CR, Easterling K, Young CD, Karwoski CJ (1993) Circadian rhythm in the visual system of the lizard Anolis carolinensis. J Biol Rhythms 8:107-124.
Takai Y, Sasaki T, Matozaki T (2001) Small GTP-binding proteins. Physiol Rev 81:153-208.

Terman M, Terman J (1985) A circadian pacemaker for visual sensitivity? Ann NY Acad Sci 453:147-161.

Tokuo H, Yunoue S, Feng L, Kimoto M, Tsuji H, Ono T, Saya H, Araki N (2001) Phosphorylation of neurofibromin by cAMP-dependent protein kinase is regulated via a cellular association of $\mathrm{N}(\mathrm{G}), \mathrm{N}(\mathrm{G})$ dimethylarginine dimethylaminohydrolase. FEBS Lett 494:48-53.

Vojtek AB, Der CJ (1998) Increasing complexity of the Ras signaling pathway. J Biol Chem 273:19925-19928.

Vossler MR, Yao H, York RD, Pan MG, Rim CS, Stork PJ (1997) cAMP activates MAP kinase and Elk-1 through a B-Raf- and Rap1-dependent pathway. Cell 89:73-82.

Wang Y, Mangel SC (1996) A circadian clock regulates rod and cone input to fish retinal cone horizontal cells. Proc Natl Acad Sci USA 93:4655-4660.

Williams JA, Su HS, Bernards A, Field J, Sehgal A (2001) A circadian output in Drosophila mediated by neurofibromatosis-1 and Ras/MAPK. Science 293:2251-2256.

Wu WQ, McGoogan JM, Cassone VM (2000) Circadian regulation of visually evoked potentials in the domestic pigeon, Columba livia. J Biol Rhythms 15:317-328.

Xu GF, Lin B, Tanaka K, Dunn D, Wood D, Gesteland R, White R, Weiss R, Tamanoi F (1990) The catalytic domain of the neurofibromatosis type 1 gene product stimulates ras GTPase and complements ira mutants of $S$. cerevisiae. Cell 63:835-841.

Zatz M (1992) Does the circadian pacemaker act through cyclic AMP to drive the melatonin rhythm in chick pineal cells? J Biol Rhythm 7:301-311. 